\title{
The Correlation between Atrial Fibrillation and Prognosis and Hemorrhagic Transformation
}

\author{
Hui Dang Wan-Qian Ge Chao-Fan Zhou Cheng-Ye Zhou \\ Department of Rehabilitation, The First Affiliated Hospital of Wenzhou Medical University, Wenzhou, China
}

\section{Keywords}

Atrial fibrillation · Acute ischemic stroke · Prognosis .

Modified Rankin Scale

\begin{abstract}
Objective: Atrial fibrillation (AF) is the recognized risk factor for hemorrhagic transformation (HT) in thrombolysis patients with acute ischemic stroke (AIS). But the impact of AF on prognosis is still controversial. In our study, we aimed to assess the relationship between AF and HT and prognosis. Methods: We assessed 184 patients diagnosed with AIS and received thrombolysis from January 2016 to October 2017. Based on the imaging results during hospitalization, the patients were divided into HT and non-HT groups in which the HT was containing 40 patients. According to the modified Rankin Scale (mRS), we divided the patients into favorable prognosis (mRS score of 0-2) and the poor (mRS score $>2$ ) after 3 and 6 months of follow-up. Our analysis included demographics, onset to treatment time, initial blood pressure, baseline National Institutes of Health Stroke Scale (NIHSS) score, HT, anticoagulants, AF, smoking, and other past history. Results: At baseline, there was a significant difference $(p<0.05)$ between the HT and non-HT groups in the level of age, hyperlipidemia, AF, NIHSS, and the application of anticoagulants. After 3 and 6 months of follow-up, we found that only NIHSS (OR 3 month $1.421,95 \%$ Cl 1.280-1.578, $p<0.001$, and $\mathrm{OR}_{6}$ month $\left.1.326,95 \% \mathrm{Cl} 1.217-1.445, p<0.001\right)$ was as-
\end{abstract}

\section{KARGER}

(c) 2019 S. Karger AG, Basel

E-Mail karger@karger.com

www.karger.com/ene sociated with prognosis instead of $\mathrm{AF}, \mathrm{HT}$, and anticoagulants. Meanwhile, patients with AF tended to be older, higher NIHSS score and less hyperlipidemia ( $p<0.05)$. Conclusion: The present study indicated that there is no significant correlation between AF and prognosis, although there is some indeed related with $\mathrm{HT}$. That was, the prognosis with AF had a similar response trend compared with the non-AF.

(c) 2019 S. Karger AG, Basel

\section{Introduction}

Atrial fibrillation (AF) is one of the most frequent arrhythmia clinically and whose patients have been associated with increased risk of acute ischemic stroke (AIS) [1, 2]. According to some researches, AF is accompanied with about $20 \%$ AIS patients, based on their hospitalization electrocardiogram $[3,4]$. Approximately $50-60 \%$ of AIS patients with AF are definitely or probably cardioemboic in origin $[5,6]$. The prior study suggested that compared with other causes, cardioembolic stroke was more severe symptoms [7], more often fatal [8], and more likely to be disabled [9]. Early thrombolysis by using recombinant tissue plasminogen activator ( $\mathrm{r}$-tPA) within $4.5 \mathrm{~h}$ after AIS onset was proved to be an effective treatment

Co-first authors: H.D. and W.-Q.G. 
and early recanalization is considered as a significant marker of this improvement $[10,11]$. However, it may increase the risk of hemorrhagic transformation (HT) just like a double-edged sword. HT refers to concomitant intracerebral hemorrhage after thrombolysis in AIS, a commonly seen complication in acute phases of cerebral infarction [12]. HT ranges from small petechiae to parenchymal hematomas. In recent years, there were numerous studies assessed that AF was associated with an increased risk of HT after AIS and may lead to severe stroke and early death [13-15]. Some researches proved that AIS patients with AF had better functional outcomes following intravenous thrombolysis than those without $\mathrm{AF}[16,17]$, others insisted that the burden of AF had some consequence on poor prognosis $[14,18,19]$.

Due to these conflicting results, we aimed to exploring the complex relationship between AF, HT, and prognosis.

\section{Material and Methods}

\section{Study Design}

We conducted a retrospective research in patients from our hospital, which consisted of all consecutive patients with AIS treated with intravenous thrombolysis between January 2016 and October 2017. The inclusion and exclusion criteria of the thrombolysis patients were the same as those in 2015AHA/ASA Statement [20]. The dose of $\mathrm{r}-\mathrm{tPA}(0.9 \mathrm{mg} / \mathrm{kg})$ was administered through an intravenous infusion (10\% injection, $1 \mathrm{~min}$ and $90 \%$ perfusion, $1 \mathrm{~h}$ ).

\section{Procedures}

We included the following covariates in our analysis: demographics, onset to treatment time (OTT), initial blood pressure, baseline National Institutes of Health Stroke Scale (NIHSS) score, history of using anticoagulants before and after stroke, hypertension, diabetes, hyperlipidemia, AF, and smoking.

\section{Covariates Assessment}

Hypertension was defined as systolic blood pressure $\geq 140 \mathrm{~mm}$ $\mathrm{Hg}(1 \mathrm{~mm} \mathrm{Hg}=0.133 \mathrm{kPa})$ and (or) diastolic blood pressure $>90 \mathrm{~mm} \mathrm{Hg}$. Diabetes disease was assigned if instant blood glucose $\geq 11.1 \mathrm{mmol} / \mathrm{L}$ or having a history of hypoglycemic drugs. Definition of hyperlipidemia was total cholesterol $>5.6 \mathrm{mmol} / \mathrm{L}$ or triglyceride $>1.7 \mathrm{mmol} / \mathrm{L}$ or low-density lipoprotein cholesterol $>3.4 \mathrm{mmol} / \mathrm{L}$ [21-23]. AF was based on their admission electrocardiogram or during the whole hospital stay. Smokers were defined as those with any history of smoking within 1 month prior to the stroke [24]. The anticoagulants include warfarin, rivaroxaban, dabigatran, and so on.

\section{Outcome Measures}

HT was defined as rebleeding in the ischemic tissue that could be observed on a computerized tomography (CT) scan during hospitalization. According to the European Cooperative Acute Stroke Study, HT was classified according to their appearance on CT into hemorrhagic infarction or parenchymal hemorrhage [25]. Most of
HT occurred within 15 days, approximately accounting for $87 \%$ [26]. CT was performed for AIS patients after $24 \mathrm{~h}$ thrombolysis and reviewed within 2 weeks in our research. If symptoms deteriorated, it was timely reviewed.

The prognosis was assessed at 3 and 6 months after stroke through face-to-face or telephone interview with patients or caregivers. Modified Rankin Scale (mRS) was the most widely used functional outcome measure in AIS. The mRS measures the degree of disability in daily activities. It is scored on an ordinal scale ranging from 0 (no symptoms) to 6 (death) [27]. In our research, favorable prognosis was considered as $\mathrm{mRS}$ score $0-2$, the rest was poor.

\section{Statistical Analysis}

Statistical analysis was performed using SPSS 21.0. Qualitative data meet normal distribution, and homogeneity of variance was analyzed and compared using the $t$ test between groups. Others were analyzed by Mann-Whitney U test. Sex, hypertension, diabetes, hyperlipemia, AF, smoking, and anticoagulants were compared using the chi-square distribution. The primary effect parameters were estimated by means of multivariate logistic regression.

In this retrospective study, only anonymized data previously acquired, as part of the patient workup or for service evaluation purposes, were used. The study proposal was reviewed locally, and further ethical review was not deemed necessary.

\section{Result}

A total of 199 consecutive AIS patients were treated with r-tPA, and 184 patients were enrolled. We excluded 15 patients due to irresistible factors such as loss of followup. The data collection itself was largely retrospective.

Demographics and baseline data of patients were shown in Table 1 . There were significant difference between HT and non-HT groups $(p<0.05)$ in the level of age, baseline NIHSS, AF, hyperlipidemia, and the application of anticoagulants (both before and after the stroke). No differences were found between the 2 groups in sex, OTT, hypertension, diabetes, smoking, and antiplatelet drugs. As shown in Table 1, although the patients with large-artery disease were predominant in the HT group, the incidence of HT was the highest in patients with cardioembolic stroke in each stroke subgroup.

Table 2 indicated the differences between age, baseline NIHSS, AF, and HT in the prognosis of 3 and 6 months $(p<0.05)$. On multivariate logistic regression analysis using above factors as the variables, only baseline NIHSS $\left(\mathrm{OR}_{3}\right.$ month $1.421,95 \%$ CI 1.280-1.578, $p<$ 0.001 , and $\mathrm{OR}_{6}$ month $1.326,95 \%$ CI $1.217-1.445, p<$ 0.001 ) was shown as an independent predictor of clinical outcome. Other parameters were not related to the shortmedium prognosis (Table 3 ). That was, the increased rate of HT after thrombolysis did not lead to a higher morbidity or mortality rate as a whole.
Eur Neurol 2019;82:9-14 DOI: $10.1159 / 00050419$
Dang/Ge/Zhou/Zhou 
Table 1. Baseline characteristics of patients according to HT status $(n=184)$

\begin{tabular}{|c|c|c|c|}
\hline Variable & $\mathrm{HT}(n=40)$ & Non-HT $(n=144)$ & $p$ value \\
\hline Age, years, median (IQR) & $72(62-78)$ & $66(58-74)$ & 0.015 \\
\hline Gender, male, $n(\%)$ & $25(40)$ & $95(144)$ & 0.683 \\
\hline OTT, min, median (IQR) & $214(173-268)$ & $219(171-259)$ & 0.856 \\
\hline SBP, mm Hg, median (IQR) & $150(137-164)$ & $147(133-161)$ & 0.238 \\
\hline DBP, mm Hg, median (IQR) & $85(77-92)$ & $83(75-91)$ & 0.421 \\
\hline Baseline NIHSS, $n(\%)$ & $9(5-17)$ & $6(3-11)$ & $<0.001$ \\
\hline Hypertension, $n(\%)$ & $37(40)$ & $130(144)$ & 0.668 \\
\hline Diabetes, $n(\%)$ & $12(40)$ & $29(144)$ & 0.185 \\
\hline Hyperlipidemia, $n(\%)$ & $17(40)$ & $90(144)$ & 0.023 \\
\hline $\mathrm{AF}, n(\%)$ & $21(40)$ & $32(144)$ & $<0.001$ \\
\hline Anticoagulant $1, n(\%)$ & $5(40)$ & $4(144)$ & 0.012 \\
\hline Anticoagulant $2, n(\%)$ & $12(40)$ & $21(144)$ & 0.025 \\
\hline Antiplatelet, $n(\%)$ & $5(40)$ & $9(144)$ & 0.19 \\
\hline Smoking, $n(\%)$ & $14(40)$ & $59(144)$ & 0.495 \\
\hline \multicolumn{4}{|l|}{ Stroke subtype, $n(\%)$} \\
\hline Large-artery disease & $15(37.5)$ & 65 & \\
\hline Cardioembolic & $10(25)$ & 17 & \\
\hline Other & $15(37.5)$ & 62 & \\
\hline
\end{tabular}

HT, hemorrhagic transformation; OTT, onset to treatment time; SBP, initial systolic blood pressure; DBP, initial diastolic blood pressure; NIHSS, National Institute of Health Stroke Scale; AF, atrial fibrillation; Anticoagulant 1, the past use of anticoagulants; Anticoagulant 2, the application of anticoagulant drugs after stroke; IQR, interquartile range.

Of all patients, 53 (28.8\%) patients presented with AF. Meanwhile, as shown in Table 4, AF patients were significantly older and more likely to have severe stroke than the non-AF.

\section{Discussion}

The present review demonstrated that comorbidity of AF was related with HT in the thrombolysis patients. Specifically, AF may increase the risk of intracerebral hemorrhage. However, it was not associated with the prognosis after 3 and 6 months, only NIHSS was the prognostic predictor for patients with AIS undergone thrombolysis.

There are many inconsistent results about the effect of AF on the prognosis of thrombolytic therapy. Based on the description by Yue et al. [28], AF may increase the risk of HT and early death and decrease the achievement of favorable outcome after thrombolysis. A recent study of 12,866 patients with AIS also confirmed similar results, suggesting that $\mathrm{HT}$ and mortality were higher in patients with AF than in non-AF group [18]. However, Sung et al. [16] found that AF was associated with favorable outcomes following thrombolysis in patients with severe AIS (baseline NIHSS $>10$ ). The probably reason was that the embolism caused by AF was unstable, and it was easier to disintegrate under the combined effect of human fibrinolysis system and thrombolytic agent. In the Virtual International Stroke Trials Archive trial, no significant association could be found between AF and overall stroke outcome [29].

As noted, large artery occlusion should be considered when NHISS is $>8$ scores because the recanalization rate after thrombolysis is low, and the long-term prognosis is not optimistic under the circumstance [30]. In Wan's research, thrombolytic therapy with a higher baseline NIHSS score is prone to deep HT and is more likely to predict a poor prognosis [31].

The following reasons may help explain the phenomenon about AF. First, the stability of cardiogenic embolism caused by AF in AIS patients was poor, and it was easy to cause secondary infarction or HT, which made the clinical symptoms worse. During the recovery period, with the application of antiplatelet or anticoagulant, the residual thrombus was more difficult to aggregate, and the impact on the prognosis may be weakened. Second, according to the research, AF was associated with a higher recanalization rate but higher risk for HT [32]. On the one hand, preferential recanalization meant that the thrombus dissolved more thoroughly and had certain 
Table 2. Baseline characteristics of patients according to mRS status $(n=184)$

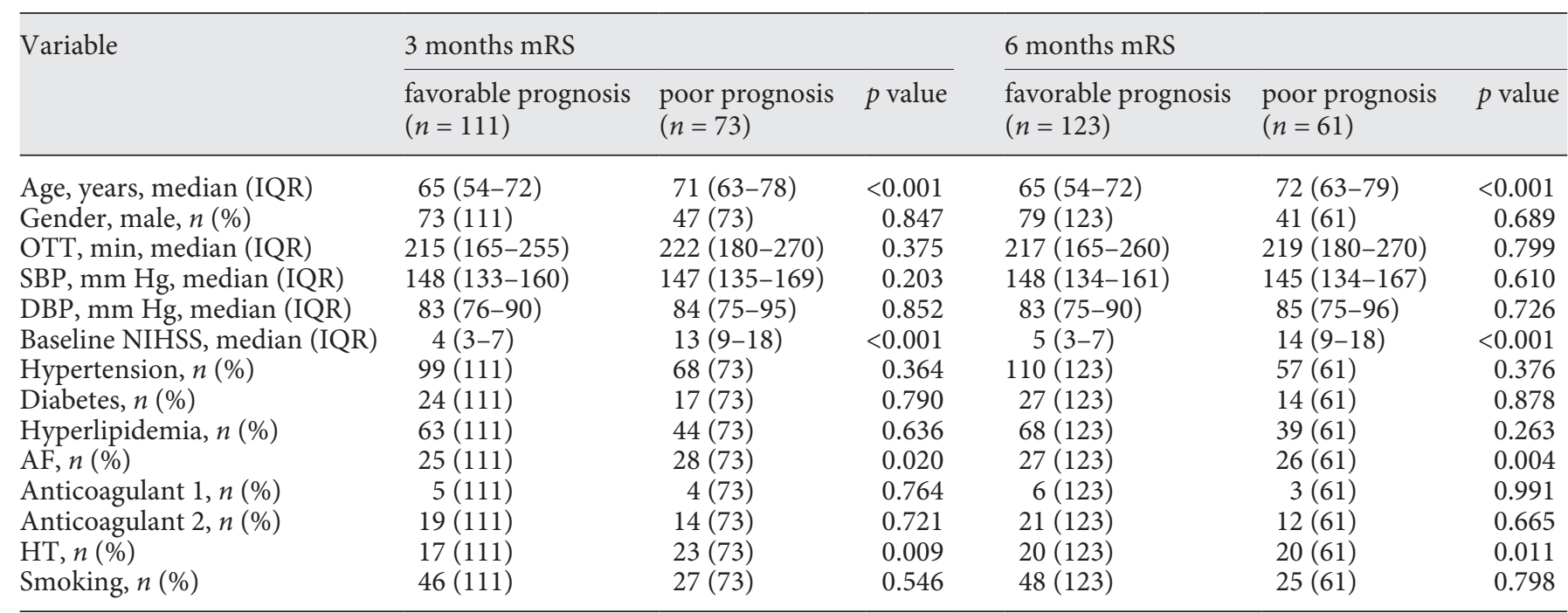

mRS, modified Rankin Scale; OTT, onset to treatment time; SBP, initial systolic blood pressure; DBP, initial diastolic blood pressure; NIHSS, National Institute of Health Stroke Scale; AF, atrial fibrillation; Anticoagulant 1, the past use of anticoagulants; Anticoagulant 2, the application of anticoagulant drugs after stroke; HT, hemorrhagic transformation; IQR, interquartile range.

Table 3. Logistic regression analysis

\begin{tabular}{|c|c|c|c|c|c|c|}
\hline \multirow[t]{2}{*}{ Variable } & \multicolumn{3}{|c|}{3 months mRS } & \multicolumn{3}{|c|}{6 months mRS } \\
\hline & OR & $95 \%$ CI & $p$ value & OR & $95 \%$ CI & $p$ value \\
\hline Age, years & 1.033 & $0.996-1.072$ & 0.085 & 1.029 & $0.992-1.068$ & 0.124 \\
\hline NIHSS & 1.421 & $1.280-1.578$ & $<0.001$ & 1.326 & $1.217-1.445$ & $<0.001$ \\
\hline $\mathrm{HT}$ & 1.249 & $0.451-3.460$ & 0.669 & 1.141 & $0.432-3.017$ & 0.790 \\
\hline $\mathrm{AF}$ & 0.762 & $0.291-1.999$ & 0.581 & 1.240 & $0.500-3.074$ & 0.643 \\
\hline Constant & 0.004 & & $<0.001$ & 0.005 & & $<0.001$ \\
\hline
\end{tabular}

mRS, modified Rankin Scale; NIHSS, baseline National Institute of Health Stroke Scale; HT, hemorrhagic transformation; AF, atrial fibrillation.

Table 4. Baseline characteristics of patients according to AF status $(n=184)$

\begin{tabular}{lccc}
\hline Variable & AF group $(n=53)$ & Non-AF group $(n=131)$ & $p$ value \\
\hline Age, years, median (IQR) & $72(65-79)$ & $65(54-73)$ & $<0.001$ \\
Gender, male, $n(\%)$ & $30(53)$ & $90(131)$ & 0.119 \\
OTT, min, median (IQR) & $211(173-253)$ & $221(170-260)$ & 0.327 \\
SBP, mm Hg, median (IQR) & $144(131-162)$ & $148(135-163)$ & 0.500 \\
DBP, mm Hg, median (IQR) & $85(77-92)$ & $83(75-91)$ & 0.374 \\
Baseline NIHSS, median (IQR) & $9(5-15)$ & $5(3-10)$ & 0.001 \\
Hypertension, $n(\%)$ & $50(53)$ & $117(131)$ & 0.286 \\
Diabete, $n(\%)$ & $10(53)$ & $83(131)$ & 0.548 \\
Hyperlipidemia, $n(\%)$ & $24(53)$ & $57(131)$ & 0.031 \\
Smoking, $n(\%)$ & $16(53)$ & & 0.094 \\
\hline
\end{tabular}

AF, atrial fibrillation; OTT, onset to treatment time; SBP, initial systolic blood pressure; DBP, initial diastolic blood pressure; NIHSS, National Institute of Health Stroke Scale; IQR, interquartile range. 
benefits for prognosis. On the other hand, as a risk factor for HT, it may also cause aggravation of clinical symptoms and dysfunction during the acute phase. The balance between the contradictions was dynamically changing. Third, in our research, we defined favorable prognosis as mRS score of $0-2$. Some studies defined it as mRS score of $0-1$ [33]. There is no definite confine about the favorable prognosis. Fourth, considering the imbalance in baseline characteristics is slightly affected for discussion of AF paradox in AIS patients.

The study also found that there was a significant difference between the AF and non-AF groups in the level of age, NIHSS, and hyperlipidemia (Table 4). According to the study by Schnabel et al. [34], in the population of over 60 years of age, the proportion of AF increased significantly for every 10 years of age, which is roughly the same as our study. Embolism caused by AF is easy to fall off repeatedly and enter the middle cerebral artery of the carotid artery system. This artery lacks abundant collateral circulation. When the blood vessel is suddenly occluded, it will lead to serious stroke events. AF seems to be a maker of baseline NIHSS rather than an independent risk factor for poor prognosis $[16,19,29]$. As the literature reported, the risk of AIS and AF both increases with age, it is anticipated that there will be greater a number of patients presenting with AIS and concomitant AF as the population ages [34]. Therefore, stroke prevention in patients with AF is particularly more important.

More than AF, demographics, previous hypertension, diabetes, hyperlipidemia, smoking, OTT, and HT were also not significantly associated with prognosis in our study. A study by Wardlaw et al. [35] found that the independent predictors of 6-month outcome were age and the severity of stroke. In addition, as noted, other factors such as glucose also play a role in determining the outcome after thrombolysis [36]. Such variables may explain the conflicts of other studies that examined the relationship between $\mathrm{AF}$ and thrombolysis treatment. The paradox may be related to the size of the study sample, the age structure of the patient, and the definition of the outcome event.

Several limitations of our analysis should be considered when interpreting our findings. First, the included patients did not provide enough information about the types of AF; therefore, we could only roughly discuss the relationship between $\mathrm{AF}$ and prognosis, without further discussion about the impact of AF subgroup. Second, we excluded patients with arterial thrombolysis to avoid possible bias using 2 methods with different rates of recanalizations and therapeutic time windows. Third, a retrospective, single center study design was used and poten-

tial biases were inevitable in the sample size. Further studies with larger sample sizes using rigorous study designs were needed to confirm the findings of our study.

\section{Conclusion}

Through this study, we have the following points to be clear. First, AIS patients with AF are significantly associated with $\mathrm{HT}$ after thrombolytic therapy. However, there was no clear correlation in the prognosis between patients without AF. This indicates that AIS patients with AF can still benefit from thrombolysis within a defined time window, but may increase the incidence of HT. Adequate understanding of outcomes of intravenous thrombolysis in AIS patients with or without AF helps us make treatment decisions. Therefore, for AIS patients with AF, intravenous thrombolysis should be given during the time window and timely reviewed CT. Second, AF and related diseases should be treated comprehensively during the treatment of the disease including necessary anticoagulant therapy. Patients in AF group are significantly higher in age and NIHSS than non-AF group. We surmises that the AIS combined with AF may lead to more severe stroke events. Therefore, we suggest that stroke prevention is more important for AIS patients with AF.

\section{Statement of Ethics}

The authors have no ethical conflicts to disclose.

\section{Disclosure Statement}

There was no sponsorship or financial support provided for this work and neither author has any conflicts of interest to disclose.

References

Eur Neurol 2019;82:9-14 DOI: $10.1159 / 000504191$
1 Wolf PA, Abbott RD, Kannel WB. Atrial fibrillation as an independent risk factor for stroke: the Framingham Study. Stroke. 1991 Aug;22(8):983-8.

2 Goldstein LB, Bushnell CD, Adams RJ, Appel LJ, Braun LT, Chaturvedi S, et al.; American Heart Association Stroke Council; Council on Cardiovascular Nursing; Council on Epidemiology and Prevention; Council for High Blood Pressure Research; Council on Peripheral Vascular Disease, and Interdisciplinary Council on QualityofCareandOutcomesResearch.Guidelines for the primary prevention of stroke: a guideline for healthcare professionals from the American Heart Association/American Stroke Association. Stroke. 2011 Feb;42(2):517-84. 
3 Kimura K, Kazui S, Minematsu K, Yamaguchi T; Japan Multicenter Stroke Investigator's Collaboration. Analysis of 16,922 patients with acute ischemic stroke and transient ischemic attack in Japan. A hospital-based prospective registration study. Cerebrovasc Dis. 2004;18(1):47-56.

4 Liao J, Khalid Z, Scallan C, Morillo C, O’Donnell M. Noninvasive cardiac monitoring for detecting paroxysmal atrial fibrillation or flutter after acute ischemic stroke: a systematic review. Stroke. 2007 Nov;38(11):2935-40.

5 Hart RG, Pearce LA, Miller VT, Anderson DC, Rothrock JF, Albers GW, et al. Cardioembolic vs. noncardioembolic strokes in atrial fibrillation: frequency and effect of antithrombotic agents in the stroke prevention in atrial fibrillation studies. Cerebrovasc Dis. 2000 Jan-Feb;10(1):39-43.

6 Benbir G, Uluduz D, Ince B, Bozluolcay M. Atherothrombotic ischemic stroke in patients with atrial fibrillation. Clin Neurol Neurosurg. 2007 Jul;109(6):485-90.

7 Jørgensen HS, Nakayama H, Reith J, Raaschou HO, Olsen TS. Acute stroke with atrial fibrillation. The Copenhagen Stroke Study. Stroke. 1996 Oct;27(10):1765-9.

8 Marini C, De Santis F, Sacco S, Russo T, Olivieri L, Totaro R, et al. Contribution of atrial fibrillation to incidence and outcome of ischemic stroke: results from a population-based study. Stroke. 2005 Jun;36(6):1115-9.

9 Lamassa M, Di Carlo A, Pracucci G, Basile AM, Trefoloni G, Vanni P, et al. Characteristics, outcome, and care of stroke associated with atrial fibrillation in Europe: data from a multicenter multinational hospital-based registry (The European Community Stroke Project). Stroke. 2001 Feb;32(2):392-8.

10 National Institute of Neurological Disorders and Stroke rt-PA Stroke Study Group. Tissue plasminogen activator for acute ischemic stroke. NEnglJMed.1995Dec;333(24):1581-7.

11 Hacke W, Kaste M, Bluhmki E, Brozman M, Dávalos A, Guidetti D, et al.; ECASS Investigators. Thrombolysis with alteplase 3 to 4.5 hours after acute ischemic stroke. N Engl J Med. 2008 Sep;359(13):1317-29.

12 Knight RA, Barker PB, Fagan SC, Li Y, Jacobs MA, Welch KM. Prediction of impending hemorrhagic transformation in ischemic stroke using magnetic resonance imaging in rats. Stroke. 1998 Jan;29(1):144-51.

13 Chen G, Wang A, Zhao X, Wang C, Liu L, Zheng $\mathrm{H}$, et al. Frequency and risk factors of spontaneous hemorrhagic transformation following ischemic stroke on the initial brain CT or MRI: data from the China National Stroke Registry (CNSR). Neurol Res. 2016 Jun;38(6):538-44.

14 Tu HT, Campbell BC, Christensen S, Desmond PM, De Silva DA, Parsons MW, et al.; EPITHET-DEFUSE Investigators. Worse stroke outcome in atrial fibrillation is explained by more severe hypoperfusion, infarct growth, and hemorrhagic transformation. Int J Stroke. 2015 Jun;10(4):534-40.
15 Saposnik G, Kapral MK, Liu Y, Hall R, O'Donnell M, Raptis S, et al.; Investigators of the Registry of the Canadian Stroke Network; Stroke Outcomes Research Canada (SORCan) Working Group. IScore: a risk score to predict death early after hospitalization for an acute ischemic stroke. Circulation. $2011 \mathrm{Feb}$; 123(7):739-49.

16 Sung SF, Chen YW, Tseng MC, Ong CT, Lin $\mathrm{HJ}$. Atrial fibrillation predicts good functional outcome following intravenous tissue plasminogen activator in patients with severe stroke. Clin Neurol Neurosurg. 2013 Jul;115(7):892-5.

17 Christou I, Alexandrov AV, Burgin WS, Wojner AW, Felberg RA, Malkoff M, et al. Timing of recanalization after tissue plasminogen activator therapy determined by transcranial doppler correlates with clinical recovery from ischemic stroke. Stroke. 2000 Aug;31(8):1812-6.

18 Saposnik G, Gladstone D, Raptis R, Zhou L, Hart RG; Investigators of the Registry of the Canadian Stroke Network (RCSN) and the Stroke Outcomes Research Canada (SORCan) Working Group. Atrial fibrillation in ischemic stroke: predicting response to thrombolysis and clinical outcomes. Stroke. 2013 Jan;44(1):99-104.

19 Seet RC, Zhang Y, Wijdicks EF, Rabinstein AA. Relationship between chronic atrial fibrillation and worse outcomes in stroke patients after intravenous thrombolysis. Arch Neurol. 2011 Nov;68(11):1454-8.

20 Demaerschalk BM, Kleindorfer DO, Adeoye OM, Demchuk AM, Fugate JE, Grotta JC, et al.; American Heart Association Stroke Council and Council on Epidemiology and Prevention. Scientific rationale for the inclusion and exclusion criteria for intravenous alteplase in acute ischemic stroke. Stroke. 2016 Feb;47(2): 581-641.

21 Geng J, Song Y, Mu Z, Xu Q, Shi G, Sun Y, et al. Early Use of Statin in Patients Treated with Alteplase for Acute Ischemic Stroke. Acta Neurochir Suppl (Wien). 2016;121:269-75.

22 Chobanian AV, Bakris GL, Black HR, Cushman WC, Green LA, Izzo JL Jr, et al.; National High Blood Pressure Education Program Coordinating Committee. The Seventh Report of the Joint National Committee on Prevention, Detection, Evaluation, and Treatment of High Blood Pressure: the JNC 7 report. JAMA. 2003 May;289(19):2560-72.

23 Alberti KG, Zimmet PZ. Definition, diagnosis and classification of diabetes mellitus and its complications. Part 1: diagnosis and classification of diabetes mellitus provisional report of a WHO consultation. Diabet Med. 1998 Jul; 15(7):539-53

24 Lee JH, Lee JY, Ahn SH, Jang MU, Oh MS, $\mathrm{Kim} \mathrm{CH}$, et al. Smoking is Not a Good Prognostic Factor following First-Ever Acute Ischemic Stroke. J Stroke. 2015 May;17(2):177-91.

25 Larrue $\mathrm{V}$, von Kummer $\mathrm{R}$ R, Müller A, Bluhmki E. Risk factors for severe hemorrhagic transformation in ischemic stroke patients treated with recombinant tissue plas- minogen activator: a secondary analysis of the European-Australasian Acute Stroke Study (ECASS II). Stroke. 2001 Feb;32(2):438-41.

26 Chen JJ, Zhang S, Zhao YH, Jin XY, Li YB, Huang LH. [Analysis on the clinical characteristics and related risk factors of patients with hemorrhagic transformation after cerebral infarction]. Zhonghua Liu Xing Bing Xue Za Zhi. 2012 Nov;33(11):1176-80.

27 Van Swieten JC, Koudstaal PJ, Visser MC, Schouten HJ, van Gijn J. Interobserver agreement for the assessment of handicap in stroke patients. Stroke. 1988 May;19(5):604-7.

28 Yue R, Li D, Yu J, Li S, Ma Y, Huang S, et al. Atrial Fibrillation is Associated With Poor Outcomes in Thrombolyzed Patients With Acute Ischemic Stroke: A Systematic Review and Meta-Analysis. Medicine (Baltimore). 2016 Mar;95(10):e3054.

29 Frank B, Fulton R, Weimar C, Shuaib A, Lees KR; VISTA Collaborators. Impact of atrial fibrillation on outcome in thrombolyzed patients with stroke: evidence from the Virtual International Stroke Trials Archive (VISTA). Stroke. 2012 Jul;43(7):1872-7.

30 Hussain SI, Zaidat OO, Fitzsimmons BF The Penumbra system for mechanical thrombectomy in endovascular acute ischemic stroke therapy. Neurology. 2012 Sep; 79(13 Suppl 1):S135-41.

31 Wan JP, Zhang S, Liu KQ, Cai X, Lou YP, Chen ZC, et al. [Risk factors of hemorrhagic transformation in different locations and its relation to clinical outcomes of patients with acute ischemic stroke following intravenous thrombolysis]. Zhejiang Da Xue Xue Bao Yi Xue Ban. 2014 Jan;43:36-42.

32 Pan XH, Liu GR, Li YC, Wang BJ, et al. Is atrial fbrillation a prognostic predictor for patients with acute ischemic stroke treated with thrombectomy? Int J Clin Exp Med. 2016; 9(3):6819-24.

33 Tsivgoulis G, Katsanos AH, Zand R, Sharma VK, Köhrmann M, Giannopoulos S, et al. Antiplatelet pretreatment and outcomes in intravenous thrombolysis for stroke: a systematic review and meta-analysis. J Neurol. 2017 Jun; 264(6):1227-35.

34 Schnabel RB, Yin X, Gona P, Larson MG, Beiser AS, McManus DD, et al. 50 year trends in atrial fibrillation prevalence, incidence, risk factors, and mortality in the Framingham Heart Study: a cohort study. Lancet. $2015 \mathrm{Jul}$; 386(9989): 154-62.

35 Wardlaw JM, Keir SL, Bastin ME, Armitage $\mathrm{PA}$, Rana AK. Is diffusion imaging appearance an independent predictor of outcome after ischemic stroke?. Neurology. 2002 Nov; 59(9):1381-7.

36 Osei E, den Hertog HM, Berkhemer OA, Fransen PS, Roos YB, Beumer D, et al.; MR CLEAN pretrial investigators. Increased admission and fasting glucose are associated with unfavorable short-term outcome after intra-arterial treatment of ischemic stroke in the MR CLEAN pretrial cohort. J Neurol Sci. 2016 Dec;371:1-5. 